\title{
CONTINUOUS STATE BRANCHING PROCESSES
}

\author{
BY JOHN LAMPERTI ${ }^{1}$
}

Communicated by Henry McKean, January 20, 1967

1. Introduction. A class of Markov processes having properties resembling those of ordinary branching processes, but with continuous instead of discrete states, was introduced by M. Jirina in [3]. Recently it has developed that these processes (assuming continuous time parameter, stationary transition probabilities, and one-dimensional state space) form precisely the class of possible limiting processes for a sequence of Galton-Watson, or simple branching, processes which have their time and space units expanding at suitable rates to infinity [5]. The purpose of this announcement is to describe the construction of the most general process of the above sort. It turns out that every such process can be obtained by a random time change from a process with stationary independent increments which cannot jump to the left. We will state these results precisely in $\$ 3$ below, and discuss some details and examples in $\$ 4$. Proofs of the main results will appear elsewhere.

2. Definitions and examples. The exact class of processes we will consider has been defined in [5], where some elementary properties and examples are also given. We repeat here only the essentials.

Definition. A 'C.B. function' is a Markov transition function on the Borel sets of $[0, \infty)$ with $P_{t}(x,[0, \infty))=1$ and such that $P_{t}(x, E)$ is jointly measurable in $t$ and $x$ for each $E$, is nontrivial in the sense that $P_{t}(x,\{0\})<1$ for some $t>0, x>0$, and that the 'branching property'

$$
P_{t}(x+y, \cdot)=P_{t}(x, \cdot) * P_{t}(y, \cdot)
$$

is satisfied for all $t, x, y \geqq 0$, where $*$ denotes convolution.

Definition. A 'C.B. process' is a Markov process on $[0, \infty)$ with right-continuous paths whose transition probabilities are given by a C.B. function.

In [5] it is shown that a C.B. function must be stochastically continuous and map the space of continuous functions on $[0, \infty]$ into itself; it is a consequence that every C.B. process is automatically strong Markov.

We will work with the spatial Laplace transforms of C.B. functions, which can be written in the form

1 This research was partially supported by the NSF. 


$$
\int_{0}^{\infty} \exp (-\lambda y) P_{t}(x, d y)=\exp \left(-x \psi_{t}(\lambda)\right), \quad \lambda \geqq 0 .
$$

The Chapman-Kolmogorov equation is then equivalent to the functional equation

$$
\psi_{t+8}(\lambda)=\psi_{t}\left(\psi_{s}(\lambda)\right) .
$$

These facts are easy consequences of (1).

Perhaps the most important examples, and certainly the best known ones, are given by the formulae

$$
\begin{aligned}
\psi_{t}(\lambda) & =\lambda \exp (\alpha t)[1-(\beta \lambda / 2 \alpha)(1-\exp (\alpha t))]^{-1}, & & \text { if } \alpha \neq 0, \\
& =\lambda[1+(\beta t \lambda / 2)]^{-1}, & & \text { if } \alpha=0 .
\end{aligned}
$$

(See [2].) The transition functions with transforms (4) correspond to diffusion processes having the (backward) Kolmogorov equations

$$
\partial \phi / \partial t=\alpha x(\partial \phi / \partial x)+(\beta x / 2)\left(\partial^{2} \phi / \partial x^{2}\right) .
$$

A somewhat more general class of processes have transforms given by

$$
\begin{aligned}
\psi_{t}(\lambda) & =\lambda \exp (\alpha t / p)\left[1-\left(\beta \lambda^{p} / 2 \alpha\right)(1-\exp (\alpha t)]^{-1 / p},\right. & & \text { if } \alpha \neq 0 \\
& =\lambda\left[1+(t \beta / 2) \lambda^{p}\right]^{-1 / p}, & & \text { if } \alpha=0
\end{aligned}
$$

where $0<p \leqq 1$. The case $\alpha=0$ is especially important, for then (6) determines the totality of limiting processes which can arise from a single Galton-Watson process as the units and the initial population size tend to infinity [4]. We will return to these examples in $\$ 4$.

3. The main theorems. The examples (5), as well as the C.B. processes with step-function paths constructed by Jirina, suggest that a C.B. function would be translation invariant, at least away from the absorbing state 0 , if it were not for the fact that the 'local speed' of the process at $x$ is not constant but proportional to $x$. It is therefore quite plausible to attempt removing this factor by means of a random time change.

Let $\left\{x_{t}\right\}$ be any C.B. process, and define $J(\omega)=\sup \left\{t: x_{t}(\omega)>0\right\}$. The functional

$$
\phi_{T}(\omega)=\int_{0}^{T} x_{u}(\omega) d u
$$

is then strictly increasing as long as $T<J$; let $T(t)(=T(t, \omega))$ be its inverse function, defined for each $t$ such that

$$
t<K(\omega)=\int_{0}^{\infty} x_{u}(\omega) d u
$$


Finally, we define a new random process $\left\{y_{t}\right\}$ by means of

$$
\begin{aligned}
v_{t} & =x_{T(t)} & & \text { if } t<K, \\
& =0 & & \text { otherwise. }
\end{aligned}
$$

From the general theory due to Volkonski (as presented in slightly more generality by Dynkin [1, Chapter 10]), it follows that $\left\{y_{t}\right\}$ is also a right-continuous strong Markov process with stationary transition probabilities.

THEOREM 1. The process $\left\{y_{t}\right\}$ defined above is a process with stationary independent increments, unable to jump to the left, which has been stopped upon the instant of first reaching state 0.

Corollary 1 (Jirina). A C.B. process of the purely-discontinuous type has increasing paths.

Corollary 2. If a C.B. process has continuous paths, it must be one of those satisfying (5).

Proof of Corollary 2. The effect of the time change is to multiply the generator of $\left\{x_{t}\right\}$ by $x^{-1}$ for $x>0$, and so the processes satisfying (5) go over into Brownian motion with a drift. Since there are no other additive processes with continuous paths and the correspondence is unique, there can be no additional C.B. diffusions beyond those of (5).

Turning from analysis to construction, we now assume that $\left\{y_{t}\right\}$ is a process with independent increments, unable to jump to the left, which has been stopped when (if) it reached $0\left(y_{0}>0\right)$. We further assume that

$$
\int_{0}^{\infty} \frac{d u}{y_{u}}=\infty \quad \text { a.s. }
$$

The only way (10) can fail occurs when $y_{t} \rightarrow \infty$ 'too fast'; it is easy to see that a sufficient condition for (10) is that the increments of the (unstopped) additive process have finite expectations. This time we set $J^{\prime}(\omega)=\left\{\sup t: y_{t}(\omega)>0\right\}$, and for $T<J^{\prime}$ define the additive functional

$$
\xi_{T}(\omega)=\int_{0}^{T} \frac{d u}{y_{u}} .
$$

This function is strictly increasing (as long as $T<J^{\prime}$ ) and its inverse will again be denoted $T(t)$. A new Markov process is then defined by 


$$
\begin{aligned}
x_{t}(\omega) & =y_{T(t)} & & \text { if } T(t)<J^{\prime} \\
& =0 & & \text { otherwise. }
\end{aligned}
$$

Theorem 2. The process $\left\{x_{t}\right\}$ constructed above is a C.B. process.

It is easy to see that the two transformations we have described are inverse to each other. Therefore a one-to-one correspondence has been established between C.B. processes and additive ones without negative jumps satisfying (10). Since the latter class can all be represented through the Levy-Khintchine formula for their characteristic functions, we may now claim to have given a construction of all (onedimensional) C.B. processes.

4. Corresponding processes. In this section we shall examine some examples of the correspondence between $\left\{x_{t}\right\}$ and $\left\{y_{t}\right\}$. Let $\left\{y_{t}\right\}$ be any suitable additive process, and suppose $y_{0}$ is a 'large' state $x$. During the initial time segment $[0, t]$, the ratio $y_{t} / y_{0}$ remains close to 1 with high probability. During this time, therefore, the corresponding C.B. process $\left\{x_{t}\right\}$ behaves as if its transition function were the same as that of $\left\{y_{t}\right\}$ except for a change of time scale by the fixed factor $x$; the interval $[0, t / x]$ for $\left\{x_{t}\right\}$ corresponds to $[0, t]$ for $\left\{y_{t}\right\}$. These considerations strongly suggest the truth of the following proposition: If $P_{t}$ and $Q_{t}$ are the transition functions of a C.B. process and the corresponding additive process, then

$$
Q_{t}(0, E)=\lim _{x \rightarrow \infty} P_{t / x}(x, E+x) .
$$

We shall apply (13) to the examples mentioned in $\$ 2$. First it is convenient to recast it by taking Laplace transforms:

$$
\begin{aligned}
\int_{-\infty}^{\infty} \exp (-\lambda y) Q_{t}(0, d y) & =\lim _{x \rightarrow \infty} \exp \left(-x \psi_{t / x}(\lambda)+\lambda x\right) \\
& =\exp (-x t c(\lambda)),
\end{aligned}
$$

where $c(\lambda)=\partial \psi_{t}(\lambda) /\left.\partial t\right|_{t=0}$. Applying (14) to the C.B. diffusions whose $\psi$ functions are given in (4), we obtain

$$
\int_{-\infty}^{\infty} \exp (-\lambda y) Q_{t}(0, d y)=\exp \left(-\alpha t \lambda+\beta t \lambda^{2} / 2_{t}\right)
$$

which is the moment generating function of a shifted normal law. Thus (as expected) $\left\{y_{t}\right\}$ in this case is Brownian motion with a drift. Treating (6) the same way, we find that the corresponding additive processes satisfy 
(16) $\int_{-\infty}^{\infty} \exp (-\lambda y) Q_{t}(0, d y)=\exp \left(-\alpha p^{-1} t \lambda+\beta(2 p)^{-1} t \lambda^{p+1}\right), \quad \lambda>0$.

These functions are the transforms of stable laws of order $p+1$, with a drift term if $\alpha \neq 0$. The formula is momentarily deceptive in that $\lambda$ is real rather than imaginary; in fact, these laws are the ones whose canonical (Levy-Khintchine) measure has no mass on $(-\infty, 0)$. In particular, the C.B. processes given by (6) with $\alpha=0$, which are the possible limits of a single Galton-Watson process in the manner studied in [4], are obtained from a drift-free, maximally unsymmetric stable process via the time change determined by (11). We will return to these examples and give further applications to the limiting theory of branching processes in a future publication.

\section{REFERENCES}

1. E. B. Dynkin, Markov processes. I, Springer, Berlin, 1965.

2. W. Feller, Diffusion processes in genetics, Proc. Second Berkeley Symposium, pp. 227-246, Univ. California Press, Berkeley, Calif., 1951.

3. M. Jirina, Stochastic branching processes with continuous state space, Czech. Math. J. 8 (1958), pp. 292-312.

4. J. W. Lamperti, Limiting distributions for branching processes, Proc. Fifth Berkeley Symposium (to appear).

5. - The limit of a sequence of branching processes, $\mathrm{Z}$. Wahrscheinlichkeitsrechnung und Verw. Gebiete.

Dartmouth College and

UNIVERSITY OF CAMBRIDGE 